\title{
EFFECT OF HOMOPANTOTHENIC ACID ON THE REFRACTORY EPILEPSY IN INFANCY ${ }^{1}$
}

\author{
TAKASHI MIZUNO, SEIICHIRO SASAKI, \\ SHUICHI WATANABE, MINORU KARAHASHI \\ AND MAYUMI KOBAYASHI ${ }^{2}$ \\ Department of Pediatrics, Faculty of Medicine, \\ Tohoku University, Sendai
}

(Received May 4, 1967)

Through clinical and experimental studies, the important function of the metabolic process of GABA (1) and related substances in the excitation and inhibition of the brain has been established. As one of the focuses of this problem at this stage, the mode of uptake of exogenous GABA and related substances by the brain might be cited (3). Although GABA was once thought to be not taken up by the brain $(1,2)$, isotopic studies using ${ }^{14} \mathrm{C}$ revealed the extreme difficulty of the uptake of GABA by the brain as compared with that of other organs (3).

For these reasons, intramedullary administration has been considered the method of choice to insure the transition of GABA and related substances into the brain in children. We have obtained favorable results by the intramedullary administration of $\gamma$-amino- $\beta$-oxybutyric acid in refractory epilepsy and cerebral palsy in infants (4). In view of the extensive organ distribution and the readiness in reaching the brain of $\mathrm{PaA}, \mathrm{HOPA}$, prepared by substituting the $\mathrm{PaA} \beta$-alanine component with GABA, was said to pass through the blood-brain barrier easily (9) and exert a favorable effect on diseases of the brain (5-8).

In the present study, we used HOPA in diseases of the brain in children and wish to report its effect in children with treatment-resistant epilepsy, on the EEG of children and mentally retarded children, with particular emphasis on its clinical effect in refractory epilepsy.

\section{EXPERIMENTAL}

\section{Test Subjects}

Fifteen children with so-called treatment-resistant epilepsy, which could not be cured by various conventional treatments, were selected. Table 1 summarizes the cases: 5 cases of nodding epilepsy, 1 case of seizure of astasia, 3 cases of absence and 6 cases of grand mal seizure, ( 3 of which were complicated by petit mal seizure), for the total of 15 cases. Most cases of refractory epilepsy in childhood were of the petit mal type.

\footnotetext{
${ }^{1}$ Following abbreviations are used: GABA, $\gamma$-aminobutyric acid; HOPA, homopantothenic acid, $\mathrm{PaA}$, pantothenic acid ; EEG, electroencephalogram.

2 水野隆, 佐々木静一郎, 渡辺修一, 唐橋 実, 小林真弓
} 
TABLE 1

Test Subject

\begin{tabular}{lc}
\hline Name of Disease & No. of Case \\
\hline Nodding epilepsy & 6 \\
Absence & 3 \\
Astasia seizure & 1 \\
Grand mal epilepsy & 6 \\
\hline
\end{tabular}

\section{Method of Investigation}

In every case selected for study, it was impossible to discontinue the anticonvulsant drug, so that HOPA was added to the daily dose. In order to accurately assess the fluctuations in the state of convulsions in response to HOPA, the patient was hospitalized, as a rule, together with the mother, who observed the number and state of convulsions of the patient before and after administration of HOPA.

The EEG was used as the method of objective evaluation of the effect. Besides the fluctuations of the epileptic waves before and after administration, the basic activities in the occipital area at rest with the eyes closed were analyzed as much as possible with an automatic frequency analyzer to study the effect of HOPA. The daily dose was $1 \mathrm{~g}$ in suckling and $1.5 \mathrm{~g}$ in infants, continued for 15 to 60 days.

\section{THERAPEUTIC RESULTS}

Table 2 and 3 summarize the dose, duration of treatment, and therapeutic effect of HOPA. In nodding epilepsy, seizures completely subsided temporarily in 2 of 5 cases, followed by a marked decrease in the number of seizures. In 3 other cases, no remarkable effect was noted on the state of seizures. It is of interest that the use of HOPA brought about a clinical effect within a very short time

TABLE 2

Therapeutic results with HOPA

\begin{tabular}{rcclll}
\hline Case No. & Age & Dose & \multicolumn{2}{c}{ Name of the disease } & Result \\
\hline & years and month & g/day & & \\
1 & 3.2 & 1.5 & Nodding epilepsy & No effect \\
2 & 0.10 & 1.0 & Nodding epilepsy & Positive effect \\
3 & 3.10 & 1.5 & Nodding epilepsy & Positive effect \\
4 & 4.4 & 1.5 & Nodding epilepsy & No effect \\
5 & 0.10 & 1.0 & Nodding epilepsy & No effect \\
6 & 1.6 & 1.0 & Nodding epilepsy & Positive effect \\
7 & 10.2 & 1.5 & Absence & Positive effect \\
8 & 8.7 & 1.5 & Absence & Positive effect \\
9 & 8.2 & 1.5 & Absence efive effect \\
10 & 6.0 & 1.5 & Astasia seizure & & No effect \\
11 & 8.7 & 1.5 & Grand mal epilepsy petit mal & Positive effect $a$ \\
12 & 8.10 & 1.5 & Grand mal epilepsy & & No effect \\
13 & 5.5 & 1.5 & Grand mal epilepsy & No effect \\
14 & 8.2 & 1.5 & Grand mal epilepsy petit mal & No effect \\
15 & 6.7 & 1.5 & Grand mal epilepsy & No effect \\
16 & 8.1 & 1.5 & Grand mal epilepsy petit mal & No effect \\
\hline
\end{tabular}

${ }^{a}$ Indicates cases in which seizures have completely disappeared. Other effective cases signify the decrease in the number of attacks. Duration of treatment was 150-160 days. 
TABLE 3

Therapeutic Results with HOPA

\begin{tabular}{lccc}
\hline \hline & Positive effect & Negative effect & Total \\
\hline Absence & 3 & 0 & 3 \\
Nodding epilepsy & 3 & 3 & 6 \\
Astasia seizure & 1 & 1 & 1 \\
Grand mal epilepsy & & 5 & 6
\end{tabular}

Positive effect signifies the decrease or disappearance of seizures. Negative effect means the complete absence of changes in the number of seizures.

(1 day) in 2 cases which showed a positive effect, while all other antiepileptic and hormonal preparations failed. This might suggest a rather rapid action, however, upon relapse a few days later, an increased dose of HOPA caused no effect, so that the effect of HOPA might be only temporary.

In all of the 3 cases of absence, a sharp decrease in the number of attacks were noted. However, a complete and sustained disappearance of the attacks was not obtained. Although the selection of cases resisting all forms of treatment and the small number of cases do not allow a definite conclusion, a sharp decrease in the number of attacks in all cases is of profound interest.

Among 6 cases of grand mal epilepsy, a positive effect was obtained in only 1 patient, who also had petit mal attacks. The effect was considered to be upon the petit mal so that HOPA did not appear to be quite as effective on grand mal.

Summarizing these results, positive effects were obtained in 6 of 15 cases, and no effect in 9 . Considering the fact that all cases selected for study was those of refractory epilepsy, such therapeutic results deserve special attention.

\section{CASE REPORT}

1. Case 1. 3 year 10 month-old male.

Diagnosis — Nodding epilepsy.

Past and Family History - Normal term birth without apparent abnormality.

Present Illness - After a normal term delivery, uneventful growth was noted until the third month. From the latter half of the third month, attacks of nodding epilepsy with flexion of the whole body like a shrimp were repeated 30 to 50 times a day. As shown in Fig. 1, ACTH-Z and all other available anticonvulsants yielded only the transient effect of making the attacks milder. Intellingence gradually deteriorated and traumatic deformity of lips due to the repeated attacks had developed by the time of hospitalization at the age of 3 years and 10 months for the purpose of ketone diet therapy.

As shown in Fig. 2, typical hypsarrhythmia was noted in EEG. Before the beginning of ketone diet therapy, HOPA was given in a trial dose of $1 \mathrm{~g}$. daily. As shown in Fig. 1, the epileptic seizures showed a sharp decrease from the next day, leaving a very mild instantaneous myoclonus once a day, representing a marked effect. However, due to an incrtanease in seizures again on the 4 th day, the dose was increased to $1.5 \mathrm{~g}$.

For 20 days after HOPA administration, the number of seizures was less than 


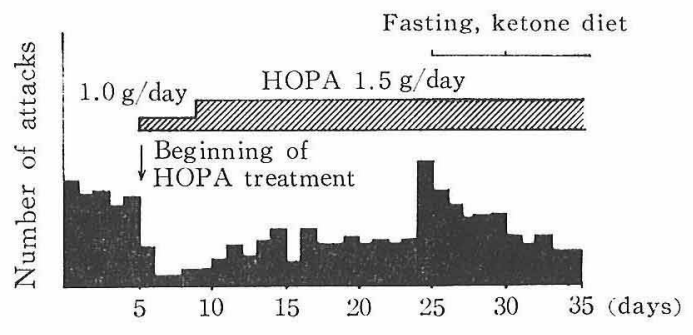

FIG. 1 Case 1

Case No. 3. Nodding epilepsy 3 years and 10 months old

Drugs used: Luminal $0.05 \mathrm{~g}$, Aleviatin $0.05 \mathrm{~g}$, Gemonil $0.15 \mathrm{~g}$, Phenetride $100 \mathrm{mg}$, ACTH-Z, Minoaleviatin, Gamibetal, and Diamox.

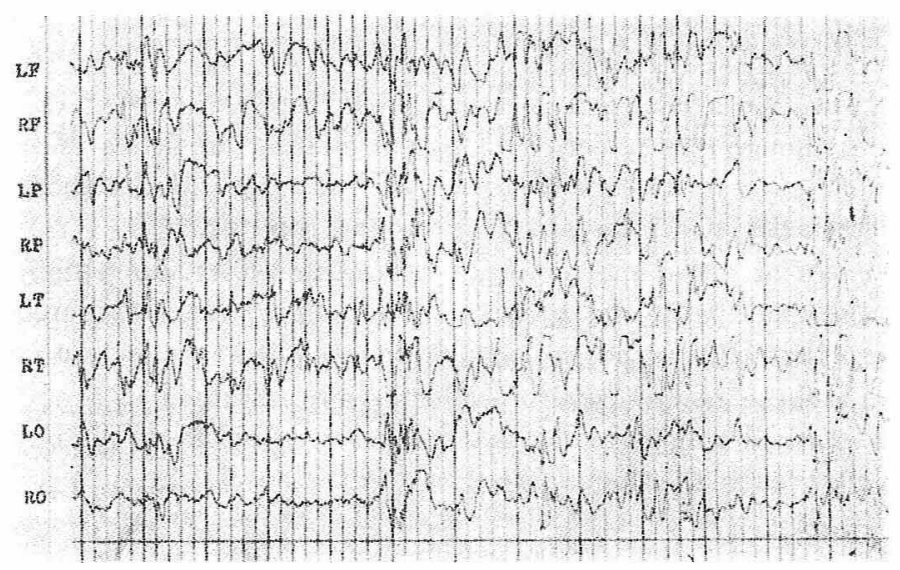

FIG. 2 Case 1

Case No. 3. 3-year and 10-month old male.

Diagnosis : Nodding epilepsy. Monopolar lead recording during a tendency to sleep. Calibration $7 \mathrm{~mm}$. Typical hypsarrhythmia is shown.

half of the level before hospitalization. However, after the 25th day of admission, the number of seizures returned to the previous level and failed to respond to an increase in dosase so that the ketone diet treatment had to be started.

2. Case 2. 8 years 7 months old female.

Diagnosis Grand mal and petit mal epilepsy.

Family History - No hereditary disease or diseases of the nervous system were found.

History of the Present Illness — Birth was normal and growth was uneventful until 2 years of age. At the age of 2 years and 10 months, the patient contracted encephalitis and her consciousness became turbid and a comatose state persisted for 10 days. A disturbance in mental development became pronounced thereafter, and grand mal epilepsy started. Subsequently, petit mal epilepsy, consisting of anterior flexion of the body accompanied by instantaneous myoclonus, developed, and intense anticonvulsant therapy was continued for a long time. 
However, the frquency of the petit mal seizures increased and the patient was hospitalized in a state of dementia. Although various function tests revealed no abnormality during admission, the EEG showed hypsarrhythmia as shown in Fig. 3. As shown in Fig. 4, most of the anticonvulsant drugs had already been tried. The oral use of HOPA, $1.5 \mathrm{~g}$ daily, gave a remarkable effect and the frequent seizures completely disappeared. The appearance of the effect within a very short time after the start of the treatment was quite impressive in this case. As the attacks of convulsion disappeared, the state of dementia improved and the consciousness became clearer.

However, gradual relapse of the petit mal was seen 2 weeks after the beginning of HOPA administration. Increase in the dosage of HOPA was without effect, and the seizures still persist after discharge.

3. Case 3. 10-year and 2-month old female

Diagnosis - Absence

Past and Family History - No particular hereditary disease or diseases of the nervous system were noted.

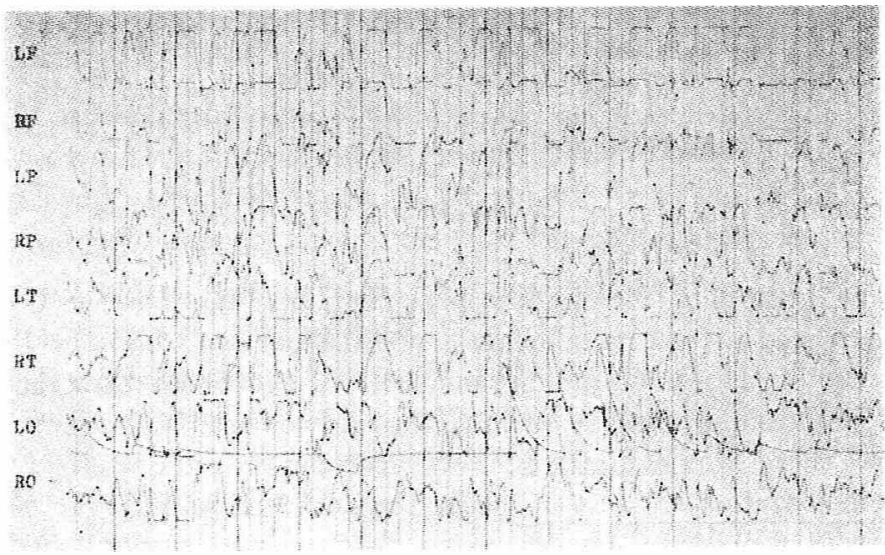

Fig. 3 Case 2

Case No. 10. 8-year and 7-month old female.

Diagnosis: Grand mal epilepsy and Petit mal seizure (myoclonus). Monopolar lead recording at the state of drowsiness. Calibration, $7 \mathrm{~mm}$. Hypsarrhythmia was seen.

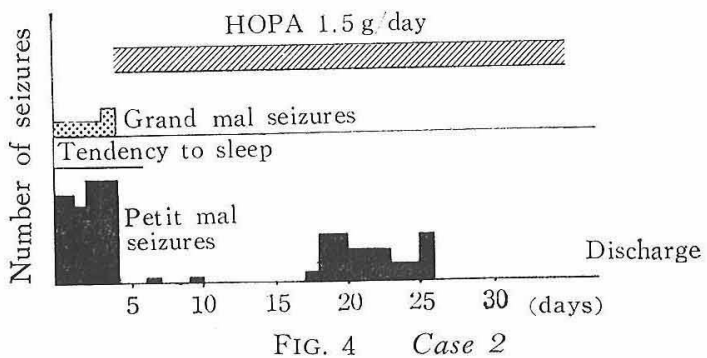

Case No. 10. Grand mal epilepsy petit mal, 8 years and 7 months old. Aleviatin $0.08 \mathrm{~g}$, Luminal $0.06 \mathrm{~g}$, Gemonil $0,3 \mathrm{~g}$ and Phenetride $100 \mathrm{mg}$. 
History of Present illness - Typical attacks of absence consisting of momentary loss of consciousness appeared at the age of 6 and treatment has been conducted ever since. As shown in Fig. 5, the use of various anticonvulsants decreased the number of attacks, leaving only several attacks a day. Although school work was above average, more attacks were noted when the patient was tired or during the nervous strain of school exhibition and $1.5 \mathrm{~g}$ of HOPA was used daily. The attacks decreased in number with no more than 1-2 brief attacks occuring a day with none at all occurring on some days. However, $3 \mathrm{c} / \mathrm{s}$ spike and wave complexes were seen in the EEG.

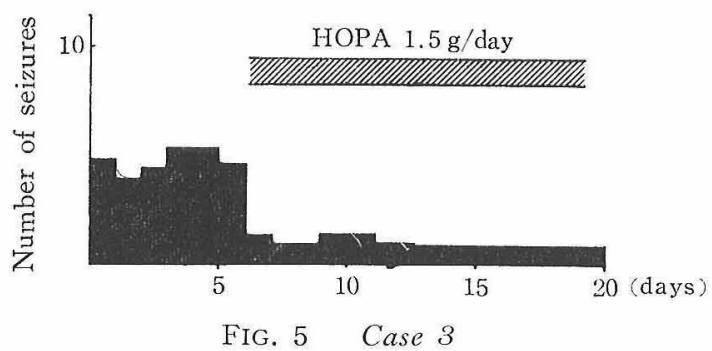

Case No. 6, 10 years and 2 months old, absence

Drugs administered, Luminal $0.1 \mathrm{~g}$, Minoaleviatin $1.0 \mathrm{~g}$, Balance $10 \mathrm{mg}$, Misolin, Gamibetal, etc,.

\section{DISCUSSION}

As stated in the beginning, unanimous agreement has been reached concerning the important role played by the metabolism of GABA on the excitatory and inhibitory system of the brain. However, no agreement has yet been made on the clinical effect of the oral administration of GABA; some found no effect at all, some maintained that there was a positive effect according to the kind of disease (10), and others demonstrated an action facilitating convulsion (11). It was also said that the inhibitory effect was due to $\gamma$-aminobutyric acid rather than GABA (11). According to our experience, the administration of GABA alone might induce convulsions or increase paroxysmal discharges in the EEG.

The HOPA preparation used in the present experiment was produced by replacing the $\beta$-alanine part of $\mathrm{PaA}$ with GABA. It is still an open question as to whether GABA is separated after oral administration to exert its effect or whether the effect of HOPA manifests itself (12). In any case, HOPA was found to be effective in animal experiments in inhibiting running cramps due to OMP, maximum cramps due to electricity and convulsions due to cardiazol (12).

In our clinical experiments on the oral administration of HOPA, it certainly appears to have an anticonvulsive effect which is not seen with GABA, in view of the fact that the action of inhibiting convulsions was seen in 6 of 15 cases of so-called treatment resistant epilepsy. A complete disappearance of the attacks was seen in some patients. The effect of HOPA in inhibiting convulsions was thus sufficiently confirmed.

However, from the results of the present study, several problems appear to be 
present in its use as a clinical antiepileptic preparation. Most important among them is that its effect is almost entirely limited to cases of petit mal. Apart from the method of selection resulting in the preponderance of cases of petit mal seizures, the predominating clinical effect of HOPA on petit mal seizures might indicate an important aspect in the mechanism of the clinical anticonvulsant effect of HOPA.

As another problem to be considered, the anticonvulsive effect was transient and even an increase in dosage could not prevent relapse. As seen in cases 1 and 2 , the relapse was seen within 2 or 3 weeks of the start of administration. It is not yet clear whether this is due to the pharmacological action of HOPA itself, or to the nature of cases resistant to all forms of treatment. Although the latter is probably responsible, treatment of fresh cases might give the answer to this problem.

Lastly, the most impressive point in the present clinical experiment with HOPA is its rapid anticonvulsive effect. In cases showing positive effects, the clinical effects made their appearance after 1 or 2 days. This is probably closely related to the ease with which HOPA passes the blood-brain barrier.

We are at present in the process of comparing the effects of the oral and intramedullary administration (4) of $\gamma$-amino- $\beta$-oxybutyric acid. It became clear that intramedullary administration gave a far more rapid and beneficial effect. In the clinical application of GABA and related substances, the method of facilitating passage through the blood-brain barrier will become one of the important facets. The effect of HOPA on the EEG will be described in a separate report.

\section{CONCLUSION}

1. In 15 cases of epilepsy resistant to various anticonvulsant drugs, homopantothenic acid (HOPA) was used to evaluate its anti-convulsant action.

2. The dose was $1.0-1.5 \mathrm{~g}$ a day for 15 to 60 days.

3. The patients selected for study were 5 with nodding epilepsy, 3 with absence, 6 with grand mal epilepsy, ( 3 of which were complicated by petit mal seizure), and one with attacks of failure to stand.

4. The clinical effect was positive (complete disappearance of the attack or a marked decrease) in 6 and negative in 9 .

5. The appearance of clinical effects was very rapid and the seizures disappeared within 1 or 2 days of the start of treatment, probably due to the greater ease with which HOPA passed the blood-brain barrier than did GABA.

6. Most of the cases with positive effects showed deviation to the petit mal group. This might represent an important aspect of the anticonvulsive effect and site of action of HOPA.

7. As far as our present experience is concerned, the anti-convulsant effect of HOPA appeared to be transient and relapse was seen after 15 to 20 days. The selection of only serious cases which resist all forms of anti-convulsant treatment might be responsible for such a phenomenon.

8. According to our experience, the oral administration of HOPA represents a method to be tried in cases of petit mal epilepsy resisting other forms of treatment.

9. Leukopenia and disturbances in liver and kidney function have not been found in patients treated with HOPA. 


\section{REFERENCES}

1. Schwerin, P., Bessman, S. P., J. Biol. Chem. 183, 37 (1950).

2. Laitha, A., Borl, S., Waelsch, H., J. Neurochem. 3, 322 (1959).

3. Kosaka, M., Conditioned Reflex 40, 22 (1963)

4. Mizuno, T., Karahashi, M., Watanabe, S., Special Issue on Conditioned Reflex: Studies on GABA, GABOB and Related Substances. 5th (1964).

5. Nishizawa, Y., Vitamins. 23, 307 (1961).

6. Hamamoto, Y., Vitamins. 27, 237 (1963).

7. Hamamoto, Y., Vitamins. 29, 162 (1964).

8. Nishizawa, Y., Vitamins. ¿7, 324 (1963).

9. Tsujino, G., Vitamins. 25, 297 (1962).

10. Otawara T., J. Japanese Pediatrics Society. 64, 2114 (1960).

11. Hayashi, T., Neurophysiology and Neurochemistory of Convulsion, Dainihontosho (1959).

12. Tsujino, G., Vitamins, 25, 304 (1962). 